\title{
Soft X-ray characterisation of the long-term properties of supergiant fast X-ray transients ${ }^{\star}$
}

\author{
P. Romano ${ }^{1}$, L. Ducci ${ }^{2,3}$, V. Mangano ${ }^{4}$, P. Esposito ${ }^{5}$, E. Bozzo ${ }^{3}$, and S. Vercellone ${ }^{1}$ \\ 1 INAF, Istituto di Astrofisica Spaziale e Fisica Cosmica - Palermo, via U. La Malfa 153, 90146 Palermo, Italy \\ e-mail: romano@ifc.inaf.it \\ 2 Institut für Astronomie und Astrophysik, Eberhard Karls Universität, Sand 1, 72076 Tübingen, Germany \\ 3 ISDC Data Center for Astrophysics, Université de Genève, 16 chemin d'Écogia, 1290 Versoix, Switzerland \\ 4 Department of Astronomy and Astrophysics, Pennsylvania State University, University Park PA 16802, USA \\ 5 INAF, Istituto di Astrofisica Spaziale e Fisica Cosmica - Milano, via E. Bassini 15, 20133 Milano, Italy
}

Received 21 March 2014 / Accepted 21 June 2014

\begin{abstract}
Context. Supergiant fast X-ray transients (SFXTs) are high mass X-ray binaries (HMXBs) that are characterised by a hard X-ray $(\geq 15 \mathrm{keV})$ flaring behaviour. These flares reach peak luminosities of $10^{36}-10^{37} \mathrm{erg} \mathrm{s}^{-1}$ and last a few hours in the hard X-rays.

Aims. We investigate the long-term properties of SFXTs by examining the soft $(0.3-10 \mathrm{keV}) \mathrm{X}$-ray emission of the three least active SFXTs in the hard X-ray and by comparing them with the remainder of the SFXT sample.

Methods. We performed the first high-sensitivity soft X-ray long-term monitoring with Swift/XRT of three relatively unexplored SFXTs, IGR J08408-4503, IGR J16328-4726, and IGR J16465-4507, whose hard X-ray duty cycles are the lowest measured among the SFXT sample. We assessed how long each source spends in each flux state and compared their properties with those of the prototypical SFXTs.

Results. The behaviour of IGR J08408-4503 and IGR J16328-4726 resembles that of other SFXTs, and it is characterised by a relatively high inactivity duty cycle (IDC) and pronounced dynamic range (DR) in the X-ray luminosity. We found $D R \sim 7400$, $I D C \sim 67 \%$ for IGR J08408-4503, and $D R \sim 750, I D C \sim 61 \%$ for IGR J16328-4726 (in all cases the IDC is given with respect to the limiting flux sensitivity of XRT, that is $1-3 \times 10^{-12} \mathrm{erg} \mathrm{cm}^{-2} \mathrm{~s}^{-1}$ ). In common with all the most extreme SFXT prototypes (IGR J17544-2619, XTE J1739-302, and IGR J16479-4514), IGR J08408-4503 shows two distinct flare populations. The first one is associated with the brightest outbursts (X-ray luminosity $L_{\mathrm{X}} \gtrsim 10^{35-36} \mathrm{erg} \mathrm{s}^{-1}$ ), while the second comprises dimmer events with typical luminosities of $L_{\mathrm{X}} \lesssim 10^{35} \mathrm{erg} \mathrm{s}^{-1}$. This double-peaked distribution of the flares as a function of the X-ray luminosity seems to be a ubiquitous feature of the extreme SFXTs. The lower DR of IGR J16328-4726 suggests that this is an intermediate SFXT. IGR J16465-4507 is characterised by a low IDC 5\% and a relatively narrow DR 40, reminiscent of classical supergiant HMXBs. The duty cycles measured with XRT are found to be comparable with those reported previously by BAT and INTEGRAL, when the higher limiting sensitivities of these instruments are taken into account and sufficiently long observational campaigns are available. By making use of these new results and those we reported previously, we prove that no clear correlation exists between the duty cycles of the SFXTs and their orbital periods.

Conclusions. The unique sensitivity and scheduling flexibility of Swift/XRT allowed us to carry out an efficient long-term monitoring of the SFXTs, following their activity across more than 4 orders of magnitude in X-ray luminosity. While it is not possible to exclude that particular distributions of the clump and wind parameters may produce double-peaked differential distributions in the X-ray luminosities of the SFXTs, the lack of a clear correlation between the duty cycles and orbital periods of these sources make it difficult to interpret their peculiar variability by only using arguments related to the properties of supergiant star winds. Our findings favour the idea that a correct interpretation of the SFXT phenomenology requires a mechanism to strongly reduce the mass accretion rate onto the compact object during most of its orbit around the companion, as proposed in a number of theoretical works.
\end{abstract}

Key words. X-rays: binaries - X-rays: individuals: IGR J0840-4503 - X-rays: individuals: IGR J16328-4726 -

X-rays: individuals: IGR J16465-4507

\section{Introduction}

Supergiant fast X-ray transients (SFXTs) are the most recently recognized (e.g. Sguera et al. 2005) class of high mass $\mathrm{X}$-ray binaries (HMXBs). They are associated with $\mathrm{OB}$ supergiant stars via IR/optical spectroscopy, and display hard X-ray $(\geq 15 \mathrm{keV})$ outbursts significantly shorter than those of typical Be/X-ray binaries, characterised by bright flares (peak

* Tables 1-4 are only available at the CDS via anonymous ftp to cdsarc.u-strasbg.fr (130.79.128.5) or via http://cdsarc.u-strasbg.fr/viz-bin/qcat?J/A+A/568/A55 luminosities of $10^{36}-10^{37} \mathrm{erg} \mathrm{s}^{-1}$ ) lasting a few hours (Sguera et al. 2005; Negueruela et al. 2006a). These bright flares are often clustered together in longer outbursts, lasting from a few hours to a few days (e.g., Romano et al. 2007, 2014; Rampy et al. 2009). Their outburst spectra in the hard X-rays resemble those of HMXBs hosting accreting neutron stars, with spectrally hard power laws combined with high-energy cut-offs, therefore it is generally assumed that all SFXTs might host a neutron star, even if pulse periods have only been measured for a few SFXTs. Since their quiescent luminosity is of the order of $\sim 10^{32} \mathrm{erg} \mathrm{s}^{-1}$ (e.g. in't Zand 2005; Bozzo et al. 2010), SFXTs display a quite 
characteristic dynamic range of 3-5 orders of magnitude. At the time of writing, the SFXT class consists of 14 sources (e.g. Romano et al. 2014, and references therein) and about as many candidates, that is, sources that have shown an SFXT-like flaring behaviour, but are still lacking a detailed classification of the optical companion. About $250 \mathrm{HMXBs}$ are currently known to reside in our Galaxy and in the Magellanic Clouds (Liu et al. 2005, 2006; Krivonos et al. 2012), which means that the SFXT population is quickly becoming not only a peculiar, but also a relevant portion of the HMXB population.

The detailed mechanisms responsible for the bright outbursts are still debated. It is generally believed that they are related to either the properties of the wind from the supergiant companion (in't Zand 2005; Walter \& Zurita Heras 2007; Negueruela et al. 2008; Sidoli et al. 2007) and/or to mechanisms that regulate or inhibit accretion (Grebenev \& Sunyaev 2007, propeller effect; Bozzo et al. 2008a, magnetic gating). Recently, a model of quasispherical accretion onto neutron stars involving hot shells of accreted material above the magnetosphere has also been proposed (Elsner \& Lamb 1977; Shakura et al. 2012, 2013).

The long-term behaviour of SFXTs - away from the prominent bright outbursts - is naturally best observed by monitoring instruments, such as the Imager on Board the INTEGRAL Satellite (IBIS, Ubertini et al. 2003) or the Burst Alert Telescope (BAT, Barthelmy et al. 2005) on board Swift (Gehrels et al. 2004), which have now gathered data spanning about a decade each. Because of their sensitivity limits, however, these monitors mostly catch only the very bright flares. The low fluxes characteristic of the states outside the bright outbursts could only be studied extensively when a highly sensitive, soft X-ray $(0.2-10 \mathrm{keV})$ telescope, the X-ray Telescope (XRT, Burrows et al. 2005) on board Swift was used in a two-year-long series of pointed observations, 3-4 days apart, during a systematic study (Sidoli et al. 2008) of IGR J16479-4514, XTE J1739-302, IGR J17544-2619, and AX J1841.0-0536 (hereafter J16479, $\mathrm{J} 1739$, J17544, and J1841, respectively). This first assessment of the time each source spends in each flux state yielded unexpected results. Not only was the time spent in outburst a small fraction (3-5\%) of the total (Romano et al. 2009), but the four sources (which we shall call initial monitoring sample hereafter) were also found to spend most of their time at mean fluxes two orders of magnitude below the bright flares, at luminosities in the $10^{33}-10^{34} \mathrm{erg} \mathrm{s}^{-1}$ range. The sources were detected in the soft X-rays for most pointings so that their inactivity duty cycle (Romano et al. 2011, and references therein) was relatively small (19-55\%), clearly at odds with with what is generally observed in the hard X-rays. These data sets also established the ubiquitous flaring activity at all intensities and all timescales probed that were consistently observed with the XRT as well as during deep pointed observations with Suzaku (e.g. Rampy et al. 2009) and XMM-Newton (e.g. Bozzo et al. 2010).

Further monitoring campaigns providing high-cadence, pointed observations for one or more orbital periods were performed on IGR J18483-0311, IGR J16418-4532, and IGR J17354-3255 (hereafter J18483, J16418, and J17354, respectively) to primarily study the effects of orbital parameters on the observed flare distributions (Romano et al. 2010, 2012b; Ducci et al. 2013). We call this latter group orbital monitoring sample.

In this paper we continue our in-depth exploration of the long-term properties of SFXTs ${ }^{1}$ with three Swift/XRT monitoring campaigns providing the first year-long high-sensitivity soft

\footnotetext{
1 Project webpage: http://www.ifc.inaf.it/sfxt/
}

X-ray coverage of IGR J08408-4503, IGR J16328-4726, and IGR J16465-4507. These three SFXTs, which we call the new monitoring sample, are probably the least studied among the SFXT population, hence the interest on each individual source, whose long term soft X-ray properties are presented here for the first time. These sources also show the lowest hard X-ray duty cycles (Ducci et al. 2010; Paizis \& Sidoli 2014). In Sects. 2 and 3 we introduce our new monitoring sample, the observing strategy, and the analysis of both the new data and the archival ones on the initial and orbital monitoring samples. In Sect. 4 we exploit the long baseline to calculate the soft X-ray inactivity duty cycle and perform an intensity-selected spectral analysis of the new monitoring sample. We also create the differential distributions of flux and luminosity for the whole SFXT sample (ten sources) searching for clues on the underlying emission mechanisms. In Sect. 5 we discuss our findings, and in Sect. 6 we summarise our results and draw our conclusions.

\section{Sample and observations}

The monitoring campaign commenced on 2011 October 20 with a focus on IGR J08408-4503 and IGR J16328-4726 for one solar year, and continued in 2013 with one year on IGR J16465-4507. Given our preliminary results at the end of 2012, we also collected more data on IGR J16328-4726 during 2013 to improve the statistics.

The transient IGR J08408-4503 (hereafter J08408) was discovered on 2006 May 15 during a $900 \mathrm{~s}$ bright flare that reached a peak flux of $250 \mathrm{mCrab}(20-40 \mathrm{keV}$, Götz et al. 2006). It was sought in archival INTEGRAL observations (Mereghetti et al. 2006), which demonstrated a recurrent transient nature, with an earlier active state in 2003. The Swift/XRT refined position (Kennea \& Campana 2006) led to an association with an O8.5Ib(f) supergiant star, HD 74194 (Masetti et al. 2006) at a distance of $\sim 3 \mathrm{kpc}$. Swift caught several bright flares from this source (Romano et al. 2013a, and references therein).

The transient IGR J16328-4726 (Bird et al. 2007, hereafter J16328) has a long history of hard X-ray activity characterised by flares lasting up to a few hours (Fiocchi et al. 2010) as observed by INTEGRAL. Swift also caught one bright flare (Romano et al. 2013b), when the source reached an unabsorbed $2-10 \mathrm{keV}$ flux of $\sim 4 \times 10^{-10} \mathrm{erg} \mathrm{cm}^{-2} \mathrm{~s}^{-1}$. The orbital period is $P_{\text {orb }}=10.076 \pm 0.003 \mathrm{~d}$ (Corbet et al. 2010), and the IR/optical counterpart is 2MASS J16323791-4723409 (Grupe et al. 2009), an O8Iafpe supergiant star (Coleiro et al. 2013).

The source IGR J16465-4507 (hereafter J16465) was discovered by INTEGRAL on 2004 September 6-7, when it averaged $8.8 \pm 0.9 \mathrm{mCrab}(18-60 \mathrm{keV})$ and subsequently showed a strong flare at $\sim 28 \mathrm{mCrab}$ on September 7 . It never triggered the $S$ wift/BAT. IGR J16465-4507 is a pulsar with $P_{\text {spin }}=$ $228 \pm 6 \mathrm{~s}$ (Lutovinov et al. 2005) and orbital period $P_{\mathrm{orb}}=$ $30.243 \pm 0.035 \mathrm{~d}$ (La Parola et al. 2010). The optical counterpart is 2MASS J16463526-4507045 (Zurita Heras \& Walter 2004) a B0.5Ib (Negueruela et al. 2007) star at a distance of about $8 \mathrm{kpc}$ (but see also Nespoli et al. 2008; Rahoui et al. 2008).

For these sources we obtained two observations per week and per object, each $1 \mathrm{ks}$ long. The XRT mode was set in AUTO for J08408 and J16328 to best exploit the XRT automatic modeswitching (Hill et al. 2004) in response to changes in the observed fluxes, and in photon-counting (PC) mode for J16465. The observation logs are reported in Tables $1-3$. We also considered data obtained while J08408, J16328, and J1841.0 were in outburst to include in our count rate distributions (Table 4). During this new monitoring campaign we collected a total 
P. Romano et al.: Soft X-ray long-term properties of SFXTs

Table 5. Summary of the Swift/XRT campaign on the three SFXTs in the new monitoring sample.

\begin{tabular}{llrrrrll}
\hline \hline Name & Nickname & $\begin{array}{r}\text { Campaign } \\
\text { start } \\
\text { (yyyy-mm-dd) }\end{array}$ & $\begin{array}{r}\text { Campaign } \\
\text { end }\end{array}$ & $\begin{array}{r}N^{a} \\
\text { (yyy-mm-dd) }\end{array}$ & $\begin{array}{r}\text { XRT net } \\
\text { exposure } \\
\text { (ks) }\end{array}$ & $\begin{array}{l}\text { Outburst } \\
\text { dates } \\
\text { (yyyy-mm-dd) }\end{array}$ & $\begin{array}{l}\text { BAT } \\
\text { trigger } \\
\text { number }\end{array}$ \\
\hline IGR J08408-4503 & J08408 & $2011-10-20$ & $2012-08-05$ & 82 & 74.4 & - & - \\
IGR J16328-4726 & J16328 & $2011-10-20$ & $2012-10-22$ & 82 & 73.5 & $2011-12-29$ & 510701 \\
& & $2013-09-02$ & $2013-10-24$ & 16 & 14.5 & - & - \\
IGR J16465-4507 & J16465 & $2013-01-20$ & $2013-09-01$ & 65 & $58.6-$ & - \\
\hline Total & & & 245 & 221.0 & \\
\hline
\end{tabular}

Notes. ${ }^{(a)}$ Number of observations obtained during the monitoring campaign.

Table 6. Inactivity duty cycle.

\begin{tabular}{|c|c|c|c|c|c|c|c|c|c|}
\hline Name (nickname) & $\begin{array}{c}\text { Limiting rate }^{a} \\
(0.2-10 \mathrm{keV}) \\
\left(10^{-3} \mathrm{c} \mathrm{s}^{-1}\right) \\
\end{array}$ & $\begin{array}{c}\text { Limiting } F^{a} \\
(2-10 \mathrm{keV}) \\
\left(10^{-12} \mathrm{erg} \mathrm{cm}^{-2} \mathrm{~s}^{-1}\right) \\
\end{array}$ & $\begin{array}{c}\text { Limiting } L^{a} \\
(2-10 \mathrm{keV}) \\
\left(10^{34} \mathrm{erg} \mathrm{s}^{-1}\right)\end{array}$ & $\begin{array}{l}\Delta T_{\Sigma} \\
(\mathrm{ks}) \\
\end{array}$ & $\begin{array}{r}P_{\text {short }} \\
(\%) \\
\end{array}$ & $\begin{array}{l}\text { IDC } \\
(\%) \\
\end{array}$ & $\begin{array}{c}\text { Rate }_{\Delta T_{\Sigma}} \\
(0.2-10 \mathrm{keV}) \\
\left(10^{-3} \mathrm{c} \mathrm{s}^{-1}\right) \\
\end{array}$ & $\begin{array}{c}\text { Distance } \\
(\mathrm{kpc}) \\
\end{array}$ & Ref. \\
\hline \multicolumn{10}{|l|}{ Initial monitoring sample } \\
\hline IGR J16479-4514 (J16479) & 16 & 2.5 & 1.1 & 29.7 & 3 & 19 & $3.1 \pm 0.5$ & 4.9 & 1 \\
\hline XTE J1739-302 (J1739) & 13 & 1.6 & 0.18 & 71.5 & 10 & 39 & $4.0 \pm 0.3$ & 2.7 & 1 \\
\hline IGR J17544-2619 (J17544) & 12 & 1.4 & 0.21 & 69.3 & 10 & 55 & $2.2 \pm 0.2$ & 3.6 & 1 \\
\hline AX J1841.0-0536 (J1841) & 13 & 1.8 & 1.6 & 26.6 & 3 & 28 & $2.4 \pm 0.4$ & $7.8 \pm 0.74$ & 2 \\
\hline \multicolumn{10}{|l|}{ New monitoring sample } \\
\hline IGR J08408-4503 (J08408) & 17 & 1.9 & 0.26 & 46.6 & 7 & 67 & $7.2 \pm 0.6$ & $3.4 \pm 0.34$ & 2 \\
\hline IGR J16328-4726 (J16328) & 14 & 2.7 & 2.5 & 47.5 & 12 & 61 & $4.0 \pm 0.4$ & $6.5 \pm 3.5$ & 3 \\
\hline IGR J16465-4507 (J16465) & 16 & 2.0 & 4.4 & 3.0 & 0 & 5 & $14.6 \pm 0.4$ & $12.7 \pm 1.3$ & 2 \\
\hline \multicolumn{10}{|l|}{ Orbital monitoring sample } \\
\hline IGR J16418-4532 (J16418) & 19 & 12.5 & 36 & 4.8 & 0 & 11 & $>9.2^{b}$ & 13 & 1 \\
\hline IGR J17354-3255 (J17354) & 14 & 2.2 & 3.3 & 7.8 & 1 & 33 & $>4.6^{b}$ & 8.5 & 4 \\
\hline IGR J18483-0311 (J18483) & 11 & 1.8 & 0.24 & 11.8 & 0 & 27 & $3.6 \pm 0.8$ & $2.83 \pm 0.05$ & 5 \\
\hline
\end{tabular}

Notes. Count rates (Col. 2) are in units of $10^{-3}$ counts s$^{-1}$ in the $0.2-10 \mathrm{keV}$ energy band. Observed fluxes (Col. 3) are in units of $10^{-12} \mathrm{erg} \mathrm{cm}^{-2} \mathrm{~s}^{-1}$ and luminosities (Col. 4) in units of $10^{34} \mathrm{erg} \mathrm{s}^{-1}$, both in the $2-10 \mathrm{keV}$ energy band. $\Delta T_{\Sigma}(\mathrm{Col} .5)$ is the sum of the exposures accumulated in all observations, each in excess of $900 \mathrm{~s}$, where only a $3 \sigma$ upper limit was achieved; $P_{\text {short }}($ Col. 6$)$ is the percentage of time lost to short observations; IDC (Col. 7, detailed in Sect. 4.2) is the inactivity duty cycle, the time each source spends undetected down to a flux limit of reported in Col. 3. Rate $_{\Delta T_{\Sigma}}$ (Col. 8, detailed in Sect. 4.3) is the observed count rate in the data for which no detections were obtained as single observations. Values for the initial monitoring sample were recalculated based on the whole length of the campaigns (Romano et al. 2011). ${ }^{(a)}$ Based on a single $900 \mathrm{~s}$ exposure. ${ }^{(b)} 3 \sigma$ upper limit.

References. (1) Rahoui et al. (2008); (2) Coleiro et al. (2013); (3) Fiocchi et al. (2013, 3-10 kpc); (4) Tomsick et al. (2009); (5) Torrejón et al. (2010).

of 245 Swift observations as part of our program for a total net XRT exposure of $\sim 221 \mathrm{ks}$ accumulated on the three sources and distributed as shown in Table 5.

\subsection{Re-analysis of the initial and orbital monitoring samples}

We considered the data on the initial monitoring sample, J16479, J1739, and J17544, collected during the first two years of monitoring (Romano et al. 2011, 2007-10-26 to 2009-11-03), and those on J1841 collected during the first year of monitoring (Romano et al. 2009, 2007-10-26 to 2008-11-15). We also considered the data on the orbital monitoring sample, J18483 (Romano et al. 2010, 2009-06-11 to 2009-07-08), J16418 (Romano et al. 2012b, 2011-02-18 to 2011-07-30), and J17354 (Ducci et al. 2013, 2012-07-18 to 2012-07-28).

We re-analysed them by using the most recent software and calibrations like the newly acquired data, as described below.

\section{Data reduction}

The XRT data were processed with standard procedures (XRTPIPELINE v0.12.6), filtering and screening criteria by using FTools (v6.13). During the monitoring campaigns the source count rates never exceeded $\sim 0.5$ count $^{-1}$, therefore only PC events (selected in grades $0-12$ ) were considered. Source events were accumulated within a circular region with a radius of 20 pixels ( 1 pixel $\left.\sim 2.36^{\prime \prime}\right)$. Background events were accumulated from an annular source-free region centred on J08408 (inner/outer radii of 100/160 pixels), and on J16465 (inner/outer radii of 80/120 pixels), and from a nearby source-free circular region (80 pixels) for $\mathrm{J} 16328$. The data obtained during outbursts to include in the count rate distributions were affected by pile-up, and were corrected for by adopting standard procedures (Vaughan et al. 2006; Romano et al. 2006). The outburst data reported in Table 4 come from the 2013 July 2 outburst for J08408 (Romano et al. 2013a), the 2009 June 10 one for J16328 (Romano et al. 2013b), and the 2012 June 14 one for J1841 (Romano et al. 2013c). For our spectral analysis, we extracted events in the same regions as those adopted to create the light curve; ancillary response files were generated with XRTMKARF to account for different extraction regions, vignetting, and PSF corrections. We used the latest spectral redistribution matrices in CALDB (20130313). For a more detailed discussion of the data analysis procedure, we refer to Romano et al. (2011, and references therein). 

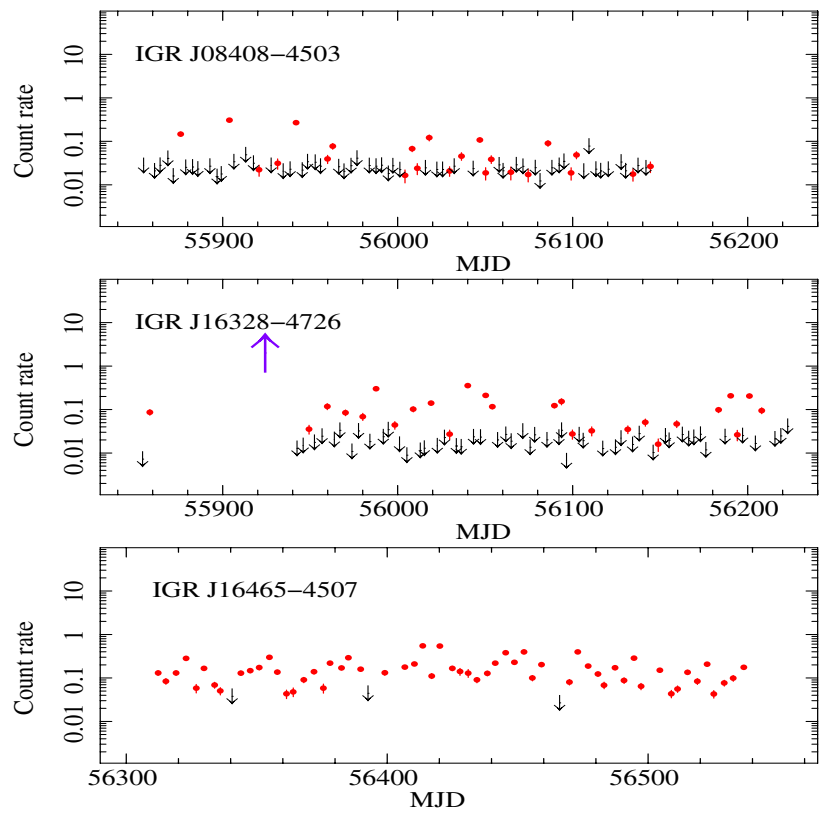

Fig. 1. Swift/XRT (0.2-10 keV) light curves. The data were collected from 2011 October 20 to 2012 October 22 and from 2013 January 20 to October 24. The (black) downward-pointing arrows are $3 \sigma$ upper limits. The upward-pointing arrow marks a flare that triggered the BAT on MJD 55924 (2011 December 29).

The BAT data of the outburst of 2011 December 29 06:39:20 UT (image trigger number 510701$)^{2}$ of J16328 were analysed using the standard BAT software within FTOOLS. The source is not detected above $\gtrsim 70 \mathrm{keV}$. The BAT mask-weighted spectrum was extracted during the first orbit of data; an energydependent systematic error vector was applied and response matrices were generated with BATDRMGEN. The spectrum was fit in the $15-70 \mathrm{keV}$ range with a simple power law, obtaining $\Gamma_{\text {BAT 2011 }}=3.0 \pm 1.0\left(\chi_{v}^{2}=1.162,37\right.$ d.o.f. $)$. The $20-50 \mathrm{keV}$ flux was $2.8 \times 10^{-10} \mathrm{erg} \mathrm{cm}^{-2} \mathrm{~s}^{-1}$.

\section{Results}

\subsection{Soft $X$-ray light curves and dynamical ranges}

The $0.2-10 \mathrm{keV}$ XRT light curves are shown in Fig. 1. They were corrected for PSF losses and vignetting and were background-subtracted. Each point in the light curves refers to the average count rate observed during each observation performed with XRT. Hereon errors on count rates are at the $1 \sigma$ level.

The dynamic range (DR), which we defined as the maximum to minimum ratio, in count rate units, is probably the simplest piece of information we can measure from the X-ray light curves. Knowing it has, however, quite a strong impact in our understanding of a source, since it can be used to distinguish (Negueruela et al. 2006b; Walter et al. 2006) between outbursts of classical supergiant HMXBs (sgHMXB, §50) and SFXTs ( 1100$)$.

For each source we calculated the observed XRT DR during this monitoring when considering individual detections, $3 \sigma$

\footnotetext{
2 See Romano et al. (2013b) for an analysis of the 2009 June 10 outburst.
}

upper limits, and the peak count rate ever observed by XRT. For J08408 we obtain a minimum DR of 25 (the highest value is $\sim 0.3$ counts $\mathrm{s}^{-1}$, the lowest a $3 \sigma$ upper limit at 0.012 counts $\left.\mathrm{s}^{-1}\right)$. The overall DR, considering the recorded outbursts (Romano et al. 2013a, and references therein) reaches then about 2000. J16328 reaches a DR in excess of 50 (the highest value is $\sim 0.3$ counts $\mathrm{s}^{-1}$, the lowest a $3 \sigma$ upper limit at 0.01 counts $\mathrm{s}^{-1}$ ). The overall DR, considering the recorded outburst (Romano et al. 2013b, maximum at $\sim 3$ counts $\mathrm{s}^{-1}$ ) then is of the order of $\sim 500$. J16465 was detected in all observations except for three, and shows a DR of 12 , the peak being 0.55 counts $\mathrm{s}^{-1}$. The DR only increases to 20 if individual $3 \sigma$ upper limits are considered (the lower is 0.026 counts s s $^{-1}$ ), as this source never triggered the BAT. By considering the detections obtained by combining all data for each source where individual observations only yielded $3 \sigma$ upper limits (see below Sect. 4.2 and Col. 8 in Table 6), the overall DR are 7400, 750, and 38, for J08408, J16328, and J16465, respectively.

\subsection{Soft $X$-ray inactivity duty cycle}

Our monitoring pace ensures a casual sampling of the X-ray light curve at a resolution of $\sim 3-4 \mathrm{~d}$ over a $\sim 1 \mathrm{yr}$ baseline, so that we can follow the procedures detailed in Romano et al. $(2009,2011)$ to calculate the percentage of time that each source spent in each flux state. Romano et al. $(2009,2011)$ defined three states, i) BAT-detected flares; ii) intermediate state (all observations yielding a firm detection, outbursts excluded); iii) "non detections" (detections with a significance below $3 \sigma$ ) with exposure in excess of $900 \mathrm{~s}$ (to account for non detections obtained during very short exposures because our observations were interrupted by a higher figure-of-merit GRB event).

The inactivity duty cycle is defined (Romano et al. 2009) as the time each source spends undetected down to a flux limit of $1-3 \times 10^{-12} \mathrm{erg} \mathrm{cm}^{-2} \mathrm{~s}^{-1}$,

IDC $=\Delta T_{\Sigma} /\left[\Delta T_{\text {tot }}\left(1-P_{\text {short }}\right)\right]$,

where $\Delta T_{\Sigma}$ is the sum of the exposures (each longer than $900 \mathrm{~s}$ ) accumulated in all observations where only a $3 \sigma$ upper limit was achieved (Table 6, Col. 5), $\Delta T_{\text {tot }}$ is the total exposure accumulated (Table 5, Col. 6), and $P_{\text {short }}$ is the fraction of time lost to short observations (exposure $<900 \mathrm{~s}$, Table 6, Col. 6). The flux limits $1-3 \times 10^{-12} \mathrm{erg} \mathrm{cm}^{-2} \mathrm{~s}^{-1}$ (Table 6, Col. 3) were obtained by converting the limiting count rates (derived from a measurement of the local background during the whole campaigns) with a count rate to flux conversion derived from the best-fit models of the low (J08408 and J16328) and medium (J16465) spectra in Table 7 (Sect. 4.3). For the initial sample we recalculated the values in Romano et al. (2011) based on the whole length of the campaigns. For the orbital monitoring sample we adopted the best fit to the first sequence described in Romano et al. (2012b) for J16418, the total spectrum in Ducci et al. (2013) for J17354, and the medium spectrum in Romano et al. (2010) for J18483. Table 6 also reports the limiting luminosities (Col. 4) and the distances adopted (Col. 9).

For the new sample we obtain that IDC $=67,61$, and $5 \%$, for J08408, J16328, and J16465, respectively (Table 6, Col. 7).

\subsection{Out-of-outburst $X$-ray spectroscopy}

We now consider the emission outside the bright outbursts. For J08408, J16328, and J16465 (totalling about 1800, 3000, and 7600 counts, respectively) we extracted the events in each observation when a detection was achieved (point ii) in Sect. 4.2), 
P. Romano et al.: Soft X-ray long-term properties of SFXTs

Table 7. XRT spectroscopy of the three SFXTs in the new monitoring sample (2011-2013 data set).

\begin{tabular}{|c|c|c|c|c|c|c|c|c|c|c|c|}
\hline Name & Spectrum & $\begin{array}{r}\text { Mean rate } \\
\left(\mathrm{c} \mathrm{s}^{-1}\right)\end{array}$ & $\left(10^{22} \mathrm{~cm}^{-2}\right)$ & $\Gamma$ & $\begin{array}{l}k T_{\mathrm{BB}} \\
(\mathrm{eV})\end{array}$ & $\begin{array}{l}R_{\mathrm{BB}} \\
(\mathrm{km})\end{array}$ & $\begin{array}{c}\text { Flux }^{a} \\
(2-10 \mathrm{keV}) \\
\left(10^{-12}\right)\end{array}$ & $\begin{array}{c}\text { Luminosity }^{b} \\
(2-10 \mathrm{keV}) \\
\left(10^{35}\right)\end{array}$ & $\chi_{v}^{2} /$ d.o.f. & $\begin{array}{c}\text { F-test } \\
\quad p\end{array}$ & Fig. \\
\hline \multirow[t]{4}{*}{ J08408 } & low & 0.05 & $0.30_{-0.30}^{+0.16}$ & $0.44_{-0.09}^{+0.13}$ & - & - & 5.6 & 0.078 & $1.7 / 57$ & & \\
\hline & low & 0.05 & $1.54_{-0.47}^{+0.50}$ & $1.02_{-0.25}^{+0.09}$ & $69_{-11}^{+14}$ & $\left(9.5_{-79}^{+56.0}\right) \times 10^{2}$ & 5.5 & 0.076 & $1.18 / 55$ & $4.4 \times 10^{-5}$ & 2 \\
\hline & very low ${ }^{d}$ & 0.009 & $0.30_{-0.00}^{+0.01}$ & $3.24_{-0.18}^{+0.18}$ & - & - & 0.09 & 0.001 & $1.47 / 194$ & & \\
\hline & very low ${ }^{d}$ & 0.009 & $0.30_{-0.30}^{+0.30}$ & $1.98_{-0.32}^{+0.31}$ & $99_{-12}^{+13}$ & $15_{-5}^{+11}$ & 0.19 & 0.003 & $1.18 / 192$ & $6.9 \times 10^{-10}$ & 2 \\
\hline \multirow[t]{3}{*}{ J16328 } & low & 0.08 & $13.56_{-1.61}^{+1.82}$ & $1.35_{-0.26}^{+0.28}$ & - & - & 16 & 1 & 110 & & 3 \\
\hline & very low ${ }^{e}$ & 0.007 & $1.54_{-1.54}^{+0.99}$ & $\begin{array}{r}0.30_{-0.22}^{+0.36} \\
\end{array}$ & - & - & 1.1 & 0.06 & $1.66 / 242$ & & \\
\hline & very low & 0.007 & $4.37_{-1.50}^{+1.74}$ & $1.01_{-0.48}^{-0.522}$ & $46_{-13}^{+20}$ & $\left(7.9_{-7.8}^{+30.0}\right) \times 10^{5}$ & 0.95 & 0.06 & $1.12 / 240$ & $3.1 \times 10^{-21}$ & 3 \\
\hline \multirow[t]{6}{*}{$\mathrm{J} 16465$} & high & $>0.25$ & $2.76_{-0.35}^{+0.39}$ & $1.05_{-015}^{+0.16}$ & - & - & 43 & 9.7 & $1.02 / 108$ & & \\
\hline & high & $>0.25$ & $3.02_{-0.38}^{-0.35}$ & $1.13_{-0.16}^{-0.15}$ & $51_{-11}^{+32}$ & $\left(2.3_{-23}^{+36.0}\right) \times 10^{5}$ & 43 & 9.8 & $0.93 / 106$ & $7.5 \times 10^{-3}$ & 4 \\
\hline & medium & {$[0.15-0.25[$} & $2.04_{-0.32}^{+0.38}$ & $0.90_{-0.15}^{+0.16}$ & - & -2.3 & 23 & 5.0 & $1.18 / 102$ & & \\
\hline & medium & {$[0.15-0.25[$} & $3.53_{-0.86}^{+0.98}$ & $1.28_{-0.24}^{+0.25}$ & $12_{-3}^{+3}$ & $\left(5.9_{-4.5}^{+19.0}\right) \times 10^{2}$ & 22 & 5.3 & $1.04 / 100$ & $1.8 \times 10^{-3}$ & 4 \\
\hline & low & $<0.15$ & $2.26_{-0.26}^{+0.86}$ & $1.42_{-0.14}^{+0.14}$ & - & - & 8.0 & 1.8 & $1.32 / 120$ & & \\
\hline & low & $<0.15$ & $2.56_{-0.30}^{-0.26}$ & $1.52_{-015}^{+0.15}$ & $55_{-9}^{+11}$ & $\left(3.4_{-2.8}^{+15.0}\right) \times 10^{4}$ & 7.9 & 1.8 & $1.17 / 118$ & $8.1 \times 10^{-4}$ & 4 \\
\hline
\end{tabular}

Notes. Uncertainties are given at a $90 \%$ confidence level for one interesting parameter. ${ }^{(a)}$ Average observed $2-10 \mathrm{keV}$ fluxes in units of $10^{-12} \mathrm{erg} \mathrm{cm}^{-2} \mathrm{~s}^{-1} \cdot{ }^{(b)}$ Average 2-10 keV luminosities in units of $10^{35} \mathrm{erg} \mathrm{s}^{-1}$ calculated by adopting the distances of Table $6 .{ }^{(c)}$ F-test probability for the addition of the blackbody component (previous line). ${ }^{(d)}$ Fit performed with the column density constrained to be higher than the one derived from optical extinction towards the optical counterpart (see Sect 4.3). ${ }^{(e)}$ Fit performed with the column density constrained to be higher than the Galactic value (see Sect 4.3).

thus effectively selecting an intermediate, non-quiescent state, and accumulated the mean spectrum. For IGR J08408-4503 and IGR J16328-4726 we call this spectrum low (see Table 7). For $\mathrm{J} 16465$, we split the events in the ranges $<0.15$ counts $\mathrm{s}^{-1}$ (low), $0.15-0.25$ counts $^{-1}$ (medium), and $>0.25$ counts $\mathrm{s}^{-1}$ (high).

Furthermore, we accumulated all data for which no detections were obtained as single exposures (point iii) in Sect. 4.2, whose combined exposure is $\Delta T_{\Sigma}$ ) and extracted spectra (very low in Table 7, 300-500 counts each), we binned them to 1 count bin $^{-1}$, and used the Cash statistics ${ }^{3}$ for the fitting. On these event lists, we performed a detection, and the resulting cumulative mean count rates are reported in Table 6 (Col. 8).

For all event lists we created exposure maps and ARFs as detailed in Romano et al. (2009). The spectra were rebinned with at least of 20 counts per energy bin, and fit in the $0.5-10 \mathrm{keV}$ (J08408 and $\mathrm{J} 16328)$ and $0.3-10 \mathrm{keV}(\mathrm{J} 16465)$ energy ranges with a simple absorbed power-law model, and an absorbed power-law model plus a blackbody (BBODYRAD) when the residuals indicated a soft X-ray excess. In that case, the F-test probability for the addition of such a component is reported in Table 7 (Col. 11) along with the fit parameters (Cols. 4-7) and their 90\% confidence level (precision) errors for one interesting parameter. We note that, given the relatively poor statistics in the soft $\mathrm{X}$-ray, this thermal component is to be considered a convenient parameterisation of the soft excess, rather than the modelling of a physical feature.

Figure 2 shows the best-fit spectra of J08408. The low spectrum of J08408 is characterised by a soft $(<2 \mathrm{keV})$ component in excess of a simple absorbed power-law continuum, as demonstrated by the trend for the absorption column density to assume an unphysical null value. Even when the absorbing column is constrained to be above the value derived from the optical extinction towards the optical counterpart HD 74194 , $0.3 \times 10^{22} \mathrm{~cm}^{-2}$ (Leyder et al. 2007), in excess of the Galactic value of $0.932 \times 10^{22} \mathrm{~cm}^{-2}$ (Kalberla et al. 2005), our fit is

\footnotetext{
3 See, http://www.swift.ac.uk/xrt_spectra/docs.php
}

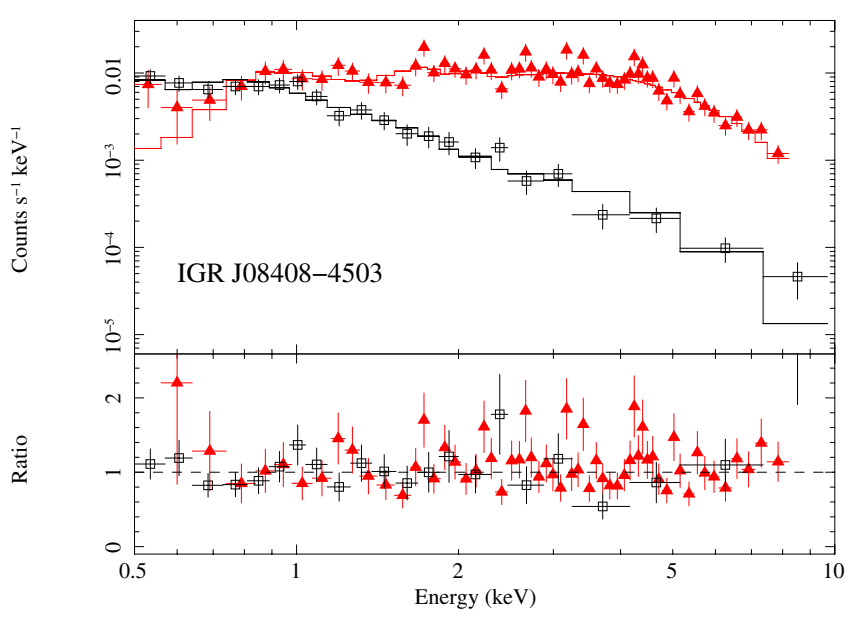

Fig. 2. Spectroscopy of the 2011-2012 campaign on J08408. Red filled triangles and black empty squares mark low and very low states. Top panel: XRT data fit with an absorbed power-law and blackbody. Bottom panel: data/model ratio.

formally unacceptable (low in Table 7). Although adding a thermal component improves the fit $\left(p=4.4 \times 10^{-5}\right)$, because of the low statistics below $\sim 2 \mathrm{keV}$, the blackbody radius is only poorly constrained. Our results are consistent with those of Bozzo et al. (2010, a 26 ks XMM-Newton observation, Col. 4 in Table 3). For the very low spectrum we obtain spectral parameters consistent with those of the fainter spectrum in Bozzo et al. (2010, Col. 2 in Table 3), although our $N_{\mathrm{H}}$ assumed the Galactic value and our derived $R_{\mathrm{BB}}$ is significantly smaller.

Figure 3 shows the spectra of J16328. The low spectrum is fit well by a simple absorbed power-law and is a factor of 3 fainter than the lowest state observed in the 2009 June 10 outburst (Romano et al. 2013b), and shows consistent spectral parameters. The addition of a soft component is only required for the very low spectrum, but the blackbody radius is unconstrained. 


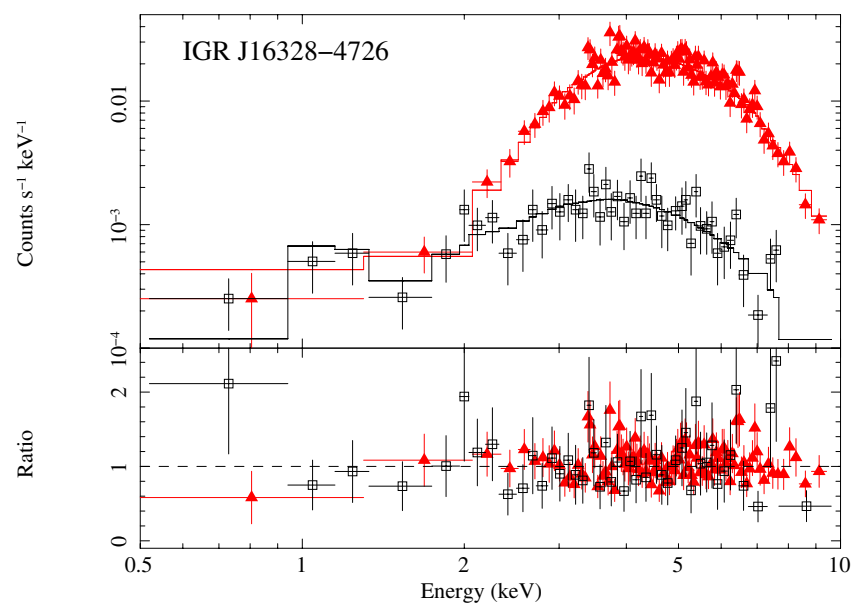

Fig. 3. Spectroscopy of the 2011-2013 campaign on J16328. Red filled triangles and black empty squares mark low and very low states, respectively. Top panel: XRT data fit with an absorbed power-law (low) and absorbed power-law plus blackbody (very low), respectively. Bottom panel: data/model ratio.

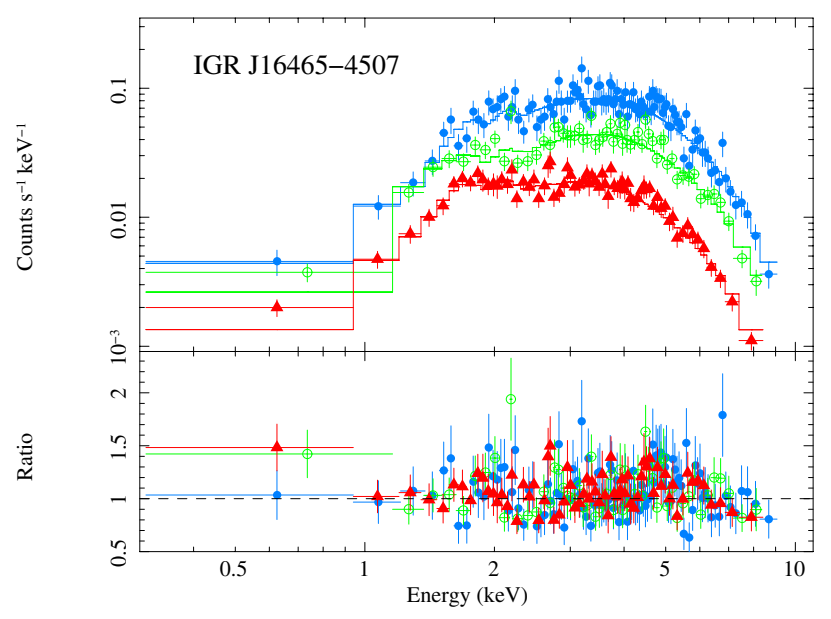

Fig. 4. Spectroscopy of the 2013 campaign on J16465. Top panel: XRT data fit with an absorbed power-law plus blackbody. Bottom panel: data/model ratio. Filled blue circles, green empty circles, and red filled triangles mark high, medium, and low states, respectively.

The spectra outside of outburst are relatively softer than those observed during the bright outbursts, as is generally observed for SFXTs when fitting the soft X-ray band data alone (Romano et al. 2013b). Our low spectrum results are consistent with those of Bozzo et al. (2012, a $22 \mathrm{ks}$ XMM-Newton observation).

Figure 4 shows the spectra of J16465. While the high spectrum is fitted adequately by a simple absorbed power-law, the residuals still show a trend for an additional soft component, therefore more fits were performed with the addition of blackbody component. The low spectrum has a similar flux to that of the spectrum observed by Morris et al. (2009, Suzaku observation), for which consistent values of absorbing column and photon index were found.

\subsection{Count rate, flux, and luminosity distributions}

Following the procedures detailed in Romano et al. (2011), we calculated the percentage of time J08408, J16328, and J16465 spent at a given flux state. To also place their behaviour in a broader context, we applied the same procedure to both the newly processed data on the initial and to the orbital monitoring samples.

Figure 5 (solid black lines) shows the differential distributions of the $2-10 \mathrm{keV}$ luminosities, drawn from the XRT light curves binned at $100 \mathrm{~s}$, after removing the observations where a detection was not achieved, ordered by length of monitoring campaign. Since the uncertainty in this conversion is dominated by those on the distance determinations, Fig. 5 also reports the flux scale $(2-10 \mathrm{keV}$, unabsorbed), as the top $x$-axis. The conversion factors for the initial monitoring sample were those calculated in Romano et al. (2011); for the orbital monitoring sample (see Sect. 2.1) those calculated in Romano et al. (2010, 2012b) and Ducci et al. (2013). For the new monitoring sample, the conversion factors were derived from the spectroscopy in Table 7. The first row shows the re-analysed data on the three SFXTs monitored for two years (Romano et al. 2011). We distinguish, in the data, those that were taken during an outburst (two for $\mathrm{J} 16479$, and three for $\mathrm{J} 1739$ and J17544) as a thin red histogram. We note that the outburst data have one bright peak in the range $\sim 10-70$ counts s ${ }^{-1}$ (corresponding to a few $10^{36}-10^{37} \mathrm{erg} \mathrm{s}^{-1}$ ), while the remainder spreads beneath the main peak of the luminosity distribution. This is due to the way the data were collected, as a BAT trigger follow-up, hence with a statistically very rich first orbit of data sometimes followed by an intense monitoring up to $\sim 10 \mathrm{ks}$ per day until the source returned to the pre-outburst levels. Figure 6 , which shows the count rate (CR) distributions in phase ${ }^{4}$ (periods in Table 8, Col. 2), further illustrates this in the panel on J16328: the hashed histogram data were taken consecutively after a bright outburst that was followed intensively.

The second row of Fig. 5 shows the four sources that were monitored for one year and never went into outburst while being monitored. To asses the overall distributions, we therefore selected one outburst and added the data as a thin red histogram. J16465 never triggered the BAT, so no data were added. For J08408 only the tail of a distribution probably peaking well below the XRT sensitivity at this binning is seen, but the outburst data clearly map another distribution, with a peak at a few counts $\mathrm{s}^{-1}$ and extending up to about $\sim 50$ counts $\mathrm{s}^{-1}$, corresponding to a few $10^{35}-10^{36} \mathrm{erg} \mathrm{s}^{-1}$. Similarly, J1841 shows a non-outburst distribution peaking at about $\sim 0.1$ counts $\mathrm{s}^{-1}$, while the outburst data peak at a few counts $\mathrm{s}^{-1}$. J16328 shows a non-outburst distribution qualitatively similar to that observed in $\mathrm{J} 1739$ and $\mathrm{J} 1841$, but the statistics do not allow us to determine whether the outburst data fall on the tail of the main distribution or if they can be distinguished from it. Based on these findings, the most probable X-ray flux for J08408 is $\$ 2 \times 10^{-12} \mathrm{erg} \mathrm{cm}^{-2} \mathrm{~s}^{-1}$ (2-10 keV, unabsorbed), for $\mathrm{J} 16328$ is $\sim 10^{-11}$ erg $\mathrm{cm}^{-2} \mathrm{~s}^{-1}$. These are about two orders of magnitude lower than the bright outbursts for these two sources. J16465 shows a well-defined distribution peaking at $\sim 0.1$ counts s$^{-1}$, corresponding to $\sim 2 \times 10^{-11} \mathrm{erg} \mathrm{cm}^{-2} \mathrm{~s}^{-1}$.

The third row of Fig. 5 shows the distributions for the three SFXTs monitored for one orbital period, none of which has XRT outburst data available. We note that J18483 triggered the BAT once on 2008 August 4, but no XRT data are available. Furthermore, while J16418 triggered the BAT four times since Swift's launch, one trigger did not have XRT follow-up, two were sub-threshold (and showed a light curve peaking at $\sim 5$ counts $\mathrm{s}^{-1}$ ), and the last had a very late follow-up, so no data with a quality matching those of the remainder of the sample are

4 The XRT light curves were first phased at the known periods, then histogrammes were created. 

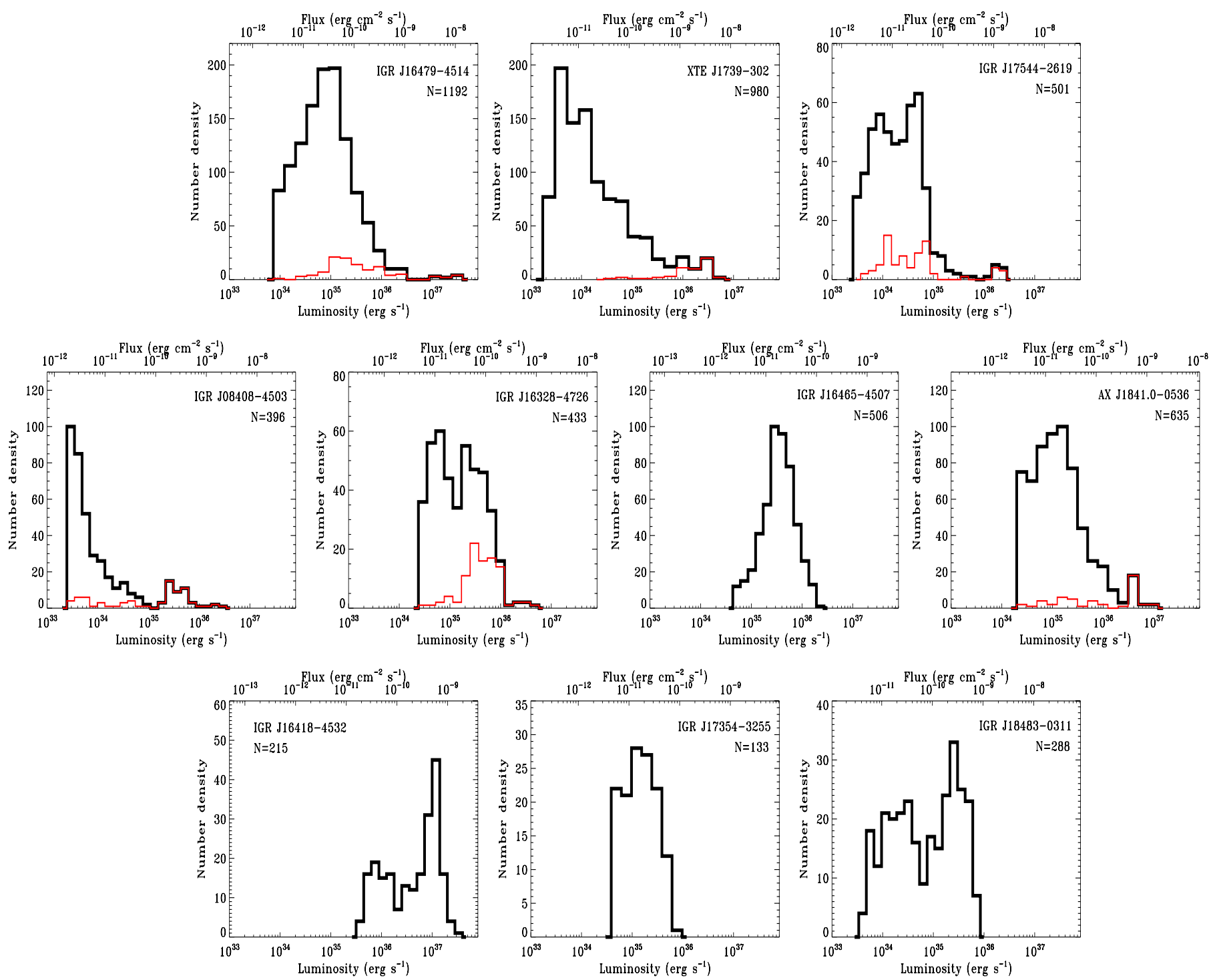

Fig. 5. Distributions of the XRT 2-10 keV luminosity (lower axis) and flux (unabsorbed, upper axis) drawn from the light curves binned at $100 \mathrm{~s}$. Top: SFXTs monitored for two years (Romano et al. 2011); the thin red histograms show the part of the data collected as outburst observations, thus including both the initial bright flare and the follow-up observations. Middle: SFXTs monitored for one year (J1841 in Romano et al. 2009); the thin red histograms show outburst observations collected outside of the monitoring campaign (one outburst per source). Bottom: SFXTs monitored for one orbital period (Romano et al. 2010; Ducci et al. 2013; Romano et al. 2012b). The sample size, $N$, is reported in each panel.

available. These data need to be taken with caution, as they were collected with an entirely different observing strategy. Indeed, while the yearly monitoring is a casual sampling of the light curves with a few points per period, these observations were collected with an intensive campaign during one or a few orbital periods. Therefore the effects of short timescale variability (variations of one order of magnitude are quite common, see Romano et al. 2010, 2012b; Ducci et al. 2013) may play the dominant role in this case.

The CR distributions in phase (Fig. 6) match the BAT light curves folded at the orbital periods reasonably well (considering the lower signal-to-noise ratio in the XRT data), as shown in Romano et al. (2014), although the eclipse throughs expected in $\mathrm{J} 16479$ and J16418 are less deep. This is a common occurrence in eclipsing HMXBs, and is discussed in terms of dust scattering by Bozzo et al. (2008b) for J16479 and by Drave et al. (2013) for J16418 as reprocessing of the intrinsic neutron star emission by the supergiant dense wind.

\section{Discussion}

\subsection{Soft X-ray long-term properties: J16465 is not an SFXT}

In this paper we report the results of a Swift/XRT monitoring of J08408, J16328, and J16465 along a baseline of more than two years and place them in the broader context of the SFXT sample.

During the campaigns only J16328 triggered the BAT and the properties of this bright flare, $\Gamma_{2011}=3 \pm 1, F_{20-50 \mathrm{keV}}=2.8 \times$ $10^{-10} \mathrm{erg} \mathrm{cm}^{-2} \mathrm{~s}^{-1}$, are consistent with those observed during the only other outburst recorded by Swift on this source $\left(\Gamma_{2009}=\right.$ $\left.2.6 \pm 0.4, F_{20-50 \mathrm{keV}}=7.1 \times 10^{-10} \mathrm{erg} \mathrm{cm}^{-2} \mathrm{~s}^{-1}\right)$. Given the lack of observed outbursts during our monitoring, and considering the outburst history of the three sources, we estimate that they spent less that $1 \%$ of their time in bright outbursts.

The main purpose of our monitoring is to exploit the unique flexibility of Swift to continue our characterisation of the longterm behaviour and emission outside the bright outbursts in SFXTs. J08408 and J16328 show activity at a level of 1-2 orders 

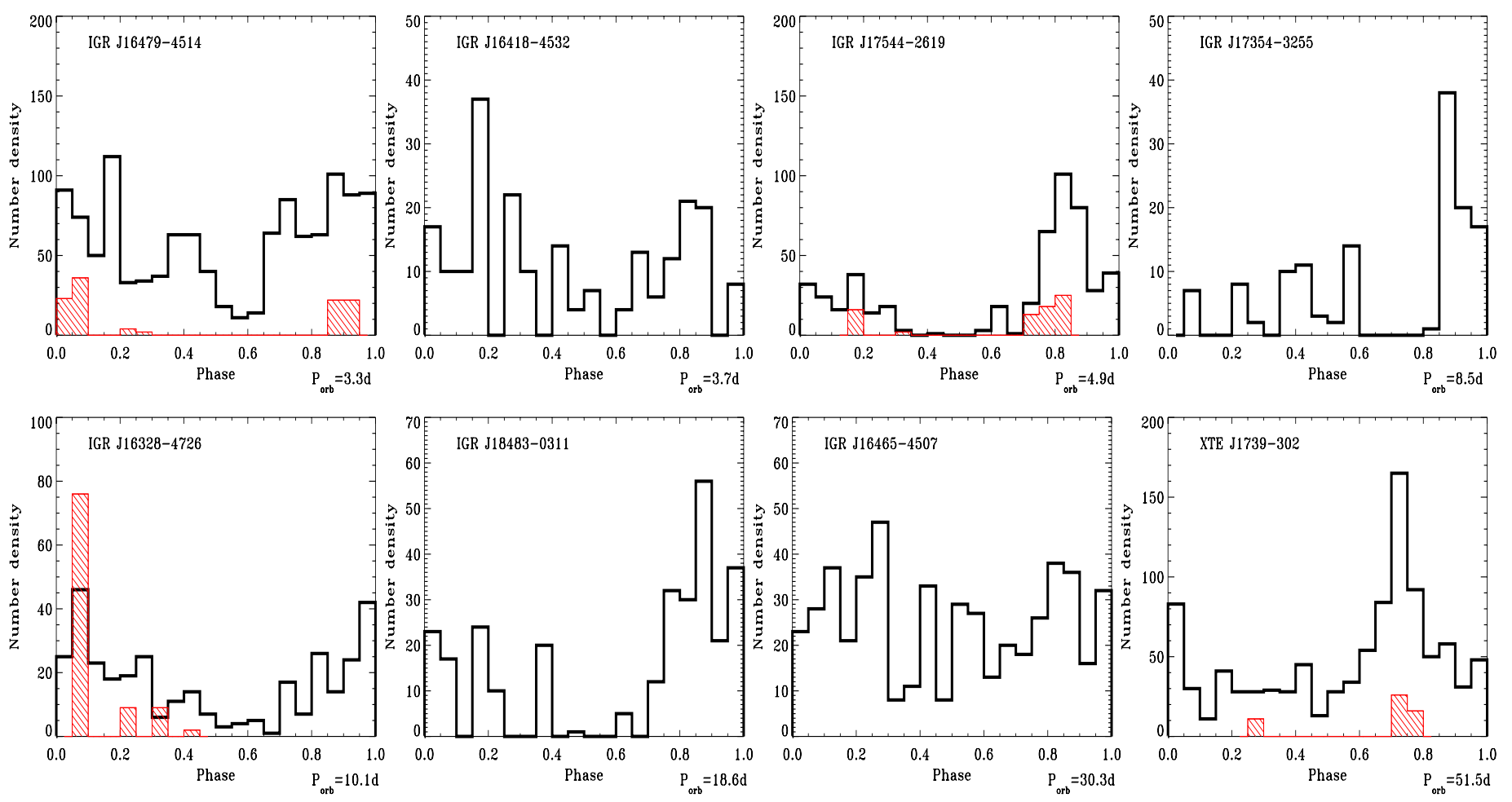

Fig. 6. Distribution of the XRT count rates $(0.3-10 \mathrm{keV})$ folded at the orbital periods, with the sources ordered by orbital period. The colour coding is the same as in Fig. 5.

Table 8. Duty cycles as a function of orbital periods.

\begin{tabular}{|c|c|c|c|c|c|c|}
\hline Name & $\begin{array}{r}\text { Orbital } \\
\text { period } \\
\text { (d) }\end{array}$ & $\begin{array}{r}\text { XRT } \\
\text { IDC }^{a} \\
(\%)\end{array}$ & $\begin{array}{c}\text { XRT } \\
\mathrm{DC}^{b} \\
(\%)\end{array}$ & $\begin{array}{c}\text { IGR } \\
\text { DC } \\
(\%)\end{array}$ & $\begin{array}{c}\text { IGR } \\
\mathrm{DC}^{d} \\
(\%)\end{array}$ & $\begin{array}{l}\text { Ref. } \\
P_{\text {orb }}\end{array}$ \\
\hline IGR J16479-4514 & 3.3193 & 19 & 1.8 & 2.8 & 2.39 & 1 \\
\hline IGR J16418 & 3.73886 & 11 & 26 & 1.3 & 0.90 & ? \\
\hline IGR J17544-2619 & 4.926 & 55 & 0.7 & 0.51 & 0.72 & 3 \\
\hline IGR J17354-3255 & 8.448 & 33 & 0.0 & - & - & 4 \\
\hline IGR J16328-4726 & 10.076 & 61 & 6.3 & - & - & 5 \\
\hline IGR J18483-0311 & 18.545 & 27 & 12 & 3.2 & 3.31 & 6 \\
\hline IGR J16465-4507 & 30.243 & 5 & 0.0 & 0.24 & 0.13 & 7 \\
\hline XTE J1739-302 & 51.47 & 39 & 4.1 & 0.83 & 0.89 & 8 \\
\hline AX J1841.0-0536 & - & 28 & 8.7 & 0.49 & 0.44 & - \\
\hline IGR J08408-4503 & - & 67 & $7 \quad 3.8$ & - & 0.16 & - \\
\hline
\end{tabular}

Notes. ${ }^{(a)}$ Inactivity duty cycle in the $0.3-10 \mathrm{keV}$ band. ${ }^{(b)}$ XRT duty cycle at the INTEGRAL sensitivity for each object (Sect. 5.2). ${ }^{(c)}$ Derived from Ducci et al. (2010), in the $20-40 \mathrm{keV}$ band. ${ }^{(d)}$ From Paizis \& Sidoli (2014), in the 17-30 keV band.

References. (1) Romano et al. (2009); (2) Drave et al. (2013); (3) Clark et al. (2009); (4) D’Aì et al. (2011); (5) Corbet et al. (2010); (6) Levine \& Corbet (2006); (7) La Parola et al. (2010); (8) Drave et al. (2010).

of magnitude lower than the bright outbursts, as previously observed for the initial monitoring sample (Romano et al. 2011, and references therein). Figure 1 shows that when the data are binned to a daily timescale, the dynamical range (25-50) of these two SFXTs is somewhat narrower than that of the initial sample, which instead showed variations spanning more than two orders of magnitude. Nevertheless, when we take into account the bright outbursts of J08408 and J16328 and the deep $3 \sigma$ upper limits obtained by combining all non-detections, their DR increases to 7400 and 750, respectively, which is typical of the SFXT population. In either case, however, they do not reach the four orders of magnitude observed in the initial sample (Fig. 1 in Romano et al. 2011).

The intermediate emission state we observed from these sources during our monitoring is characterised by non-thermal emission (hence accretion onto the compact object) following the previously observed harder-when-brighter trend (e.g. Romano et al. 2011), as well as by a soft excess whose strength becomes dominant in the very low spectra. We note that the addition of thermal components similar to those observed in other HMXBs (e.g. Hickox et al. 2004; van der Meer et al. 2005), and in particular in J08408 (Bozzo et al. 2010), improves the fit but, because of the low statistics below $\sim 2 \mathrm{keV}$, the parameters are often poorly constrained or unconstrained.

Our observations of J08408 and J16328 show that this intermediate state is characterised by soft X-ray flux variability observed on timescales of a few hundred seconds, as also observed in the initial and orbital monitoring samples, which is generally explained in terms of the clumpiness of the wind of the supergiant companion (e.g. Walter \& Zurita Heras 2007).

For J16465, La Parola et al. (2010) reported that the BAT data show a narrow DR ( $<10$ in the $15-50 \mathrm{keV}$ band) and no flaring activity, and suggested this source is a faint supergiant HMXB, probably fed by a rather homogeneous wind, and is not an SFXT. Romano et al. (2014) also reported a very scarce activity (detections in excess of $5 \sigma$ ) in the first 100 months of BAT data, and no outbursts in nine years of Swift operations. However, more outbursts were reported by Clark et al. (2010), who instead classified this source as intermediate, and by Ducci et al. (2010). Bearing this in mind, and in light of our soft X-ray findings, we discuss J16465 separately from the other two sources in the new monitoring sample. The results of the intensity-selected soft X-ray spectroscopy of this source (Table 7) can be directly compared with those on J16479 (Table 8 of Romano et al. 2011), which shows comparable luminosity levels in the high, medium, and low spectra. In particular, 
we note both the consistency of the photon indices when a simple absorbed power-law model is adopted, and the general trend for harder-when-brighter emission, which is commonly observed in SFXTs. From this point of view, then, the spectral behaviour of J16465 is consistent with what we expect from the SFXT (as well as, of course, from the general sgHMXB) population.

However, the XRT overall DR is below 40, as is typical of the general HMXB population, and not of SFXTs, and very little variability is observed in data binned at timescales of $100 \mathrm{~s}$, for which a $D R \lesssim 5$ is observed within one orbit. Furthermore, the histogram of the observed CR is single-peaked and, differently from the other SFXTs, does not show a secondary peak corresponding to the outburst data. The steepness of the distribution wings indicates that no emission is observed in excess of $\sim 1$ count $^{-1}$. The full-width at zero intensity of the distribution is considerably less than two decades, while the other SFXTs exceed three orders of magnitude. Finally, the measured IDC $(5 \%)$ is at the very low end of the observed distribution in SFXTs, since the lowest value is that of J16418. To all intents and purposes, especially considering that this source is not particularly absorbed and its distance is at the high end of the SFXT distribution, J16465 is a persistent source in the XRT. The current soft X-ray data seem to point toward a Vela X-1-like source (Kreykenbohm et al. 2008), and not to an SFXT.

We note that the discordant behaviour of J16465 with respect to that of the remainder of the INTEGRAL SFXT sample was also reported by Lutovinov et al. (2013). Within their proposed model for wind-fed HMXBs hosting a neutron star, they produced a theoretical hard X-ray luminosity vs. orbital period $\left(P_{\text {orb }}-L_{\mathrm{X}}\right)$ diagram with which both normal wind-fed HMXBs and SFXTs are compared. While normal wind-fed HMXBs are observed to lie above the lower limit of luminosity allowed for a given period $\left(L_{\mathrm{X}} \sim P_{\mathrm{orb}}^{-4 / 3}\right)$, the SFXT population shows a median luminosity beneath this curve. Therefore, the flaring observed in SFXTs can be explained within this context, provided that some mechanism, such as magnetic arrest, inhibits accretion. In the $P_{\text {orb }}-L_{\mathrm{X}}$ diagram, however, IGR J16465-4507 lies in the same allowed area as normal wind-fed HMXBs. We note that our luminosity distribution (Fig. 5) for this source reaches down to $\gtrsim 4 \times 10^{34} \mathrm{erg} \mathrm{s}^{-1}$, and the XRT lowest detection (obtained by summing all $3 \sigma$ upper limits, see Sect 4.1 ) corresponds to $\sim 3.8 \times 10^{34} \mathrm{erg} \mathrm{s}^{-1}$. These values are much closer to the $L_{\mathrm{X}} \sim P_{\text {orb }}^{-4 / 3}$ limit than the INTEGRAL data, therefore it is possible that deeper observations of this source and a better determination of the distance ${ }^{5}$ might just make it cross out of the allowed ranges in the $P_{\text {orb }}-L_{\mathrm{X}}$ diagram, which occur at $\sim 10^{34} \mathrm{erg} \mathrm{s}^{-1}$ for a period of about $30 \mathrm{~d}$ (see Fig. 10 of Lutovinov et al. 2013), like the remainder of the SFXT sample. If that were not the case, however, this would add to the evidence that IGR J16465-4507 is indeed a normal wind-fed HMXB, and not an SFXT.

\subsection{Duty cycles and orbital geometry}

A long-standing question in the SFXT field is whether the duty cycle is related to the orbital parameters, the period in primis. If the dominant source of X-ray variability in SFXTs were the properties of the binary geometry and inhomogeneity of the stellar wind from the donor star, as proposed in the clumpy wind models (e.g. in't Zand 2005; Negueruela et al. 2008; Walter \& Zurita Heras 2007), we could expect generally larger IDCs for

\footnotetext{
5 Note that the luminosity values become $\sim 2 \times 10^{34} \mathrm{erg} \mathrm{s}^{-1}$ at the distance of $9.4 \mathrm{kpc}$ adopted by Lutovinov et al. (2013).
}

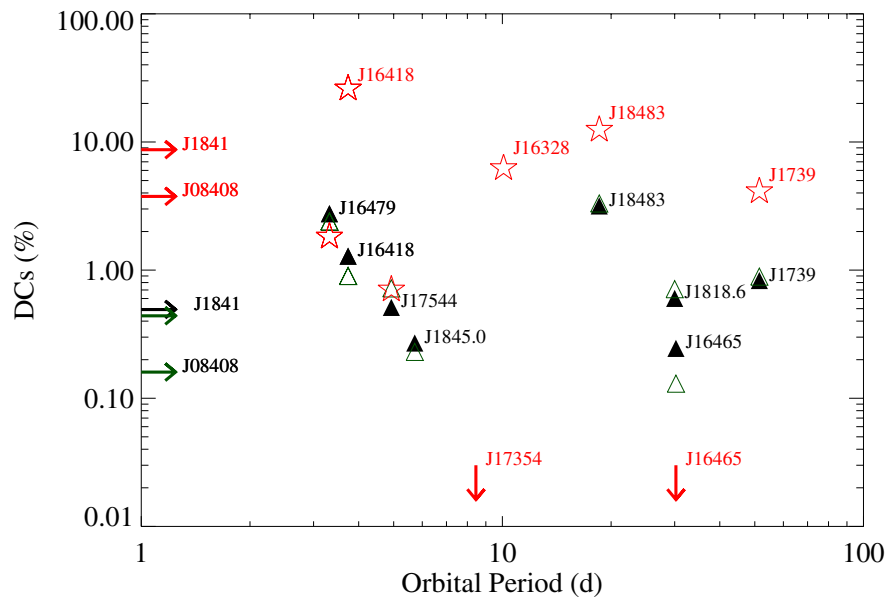

Fig. 7. INTEGRAL-based duty cycles from Ducci et al. (2010, black filled triangles) and Paizis \& Sidoli (2014, green empty triangles), and XRT duty cycle at the INTEGRAL sensitivity (red empty stars, Sect. 5.2). The downward-pointing arrows are consistent with 0 . The right-pointing arrows at $P_{\text {orb }}=1$ are for sources lacking an orbital period.

longer orbital periods. We can now address this question with high-sensitivity data.

We defined the inactivity duty cycle (Romano et al. 2009) as the time each source spends undetected down to a flux limit of $1-3 \times 10^{-12} \mathrm{erg} \mathrm{cm}^{-2} \mathrm{~s}^{-1}$ (see Table 6), thus exploiting the higher XRT sensitivity when compared with hard X-ray detec-

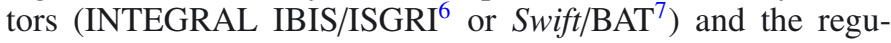
lar sampling of our monitoring campaigns. The initial sample showed that these sources were actually active for the great majority of time when inspected at fluxes as low as those achievable with the high sensitivity of XRT. Similarly, our re-analysis of the data on the orbital monitoring sample shows very low IDCs (11 to 33\%). The IDCs of J08408 and J16328 (67 and 61\%, respectively) are by far the highest of the SFXT sample, as these sources are not detected for the majority of time. In contrast, J16465 has an IDC of 5\%, which is consistent with the source being persistent.

It is interesting to compare our IDC with the DC estimated from INTEGRAL, whose instruments have a lower sensitivity for fainter luminosity states of the SFXTs, but which can provide longer-term observations. The INTEGRAL IBIS/ISGRI data are presented in Ducci et al. (2010, seven objects in common with our sample; Table 8, Col. 5), for which the most active sources are $\mathrm{J} 18483$ and $\mathrm{J} 16479$, and the least active is J16465. In Ducci et al. (2010) the duty cycle (INTEGRAL DC) is defined as the ratio of the time the sources are detected in excess of $5 \sigma$ and the total exposure time in the $20-40 \mathrm{keV}$ band. Similar results are found when the INTEGRAL DCs are drawn from the recent work of Paizis \& Sidoli (2014, eight objects in common; Table 8, Col. 6). Figure 7 shows them as as function of the orbital period. We note that our IDC is generally anti-correlated with the INTEGRAL DCs, with the notable exception of J16465.

Both the XRT IDC and the INTEGRAL DC are based on the instrumental sensitivity in the detector band. What follows is an attempt to overcome these biases. We define an XRT luminosity-based duty cycle (XRTDC) as the percentage of time

6 ISGRI reaches a sensitivity (Paizis et al. 2013) of $20 \mathrm{mCrab}$ in the $17-60 \mathrm{keV}$ band, at the $5 \sigma$ level for 1 pointing ( $2 \mathrm{ks})$.

7 BAT reaches a sensitivity (Krimm et al. 2013) of $12.9 \mathrm{mCrab}$ in the $15-50 \mathrm{keV}$ band, at $1 \sigma$ level for one orbit ( 1 ks). 


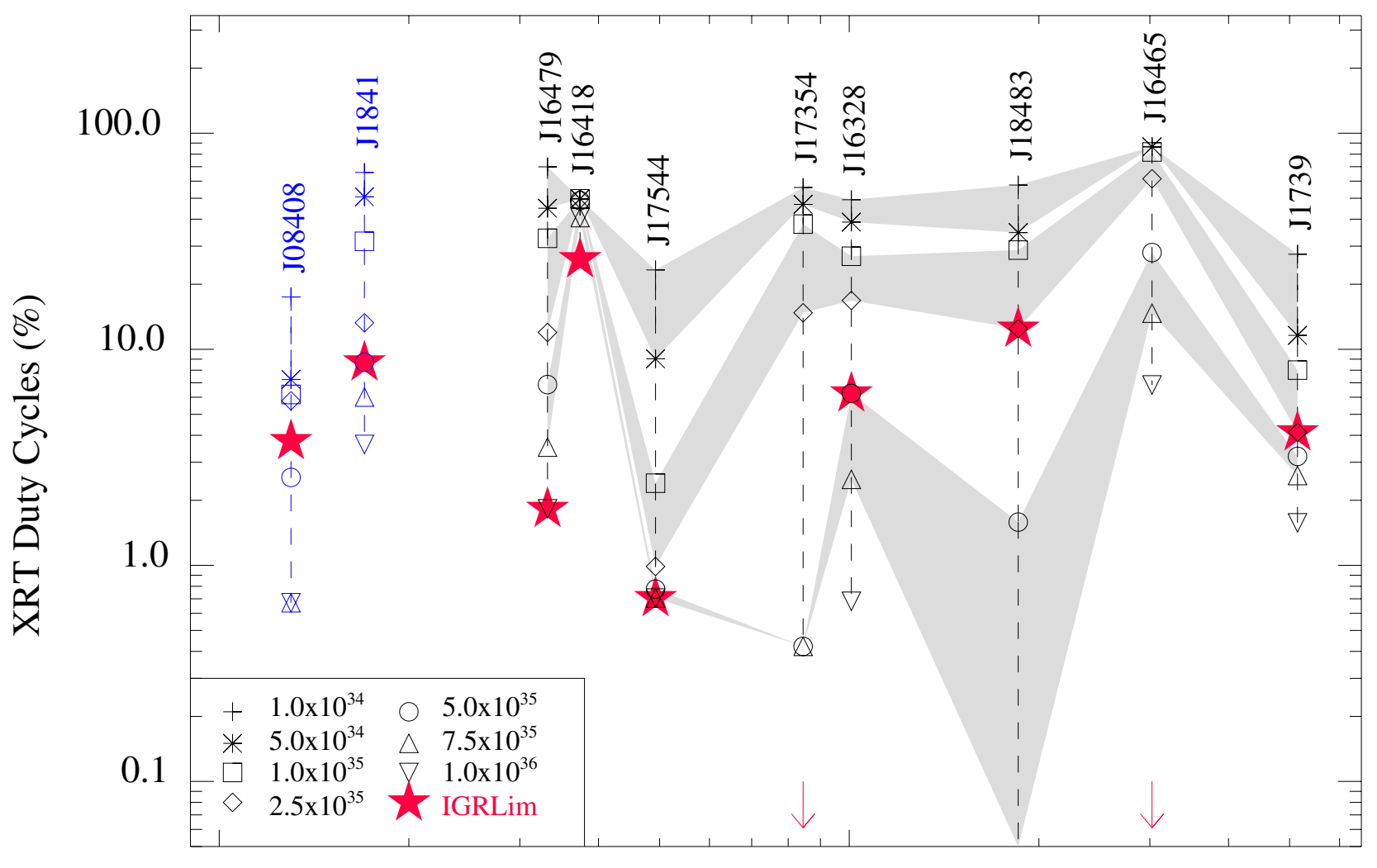

Orbital Period (d)

Fig. 8. XRTDC (2-10 kev) as a function of orbital period and for a range of $2-10 \mathrm{keV}$ luminosities (see legend, in units of erg s $\mathrm{s}^{-1}$ ) in black. All values are also reported for J08408 and J1841, which lack an orbital period measurement, as blue data points at an arbitrary orbital period shorter than two days. Only points above $0.1 \%$ are shown. The shaded areas mark the loci of XRTDC defined with contiguous luminosities. The red filled stars represent the XRT DC at the INTEGRAL sensitivity for each object (the downward-pointing arrows are consistent with 0).

the source spends above a given luminosity, and we considered several luminosities in the range $L_{2-10 \mathrm{keV}}=10^{34}-10^{36} \mathrm{erg} \mathrm{s}^{-1}$. Figure 8 shows the XRTDC as a function of the orbital period. We find that, clearly, the definition of duty cycle is strongly dependent on the luminosity assumed as lower limit for the calculation.

In particular, we can also consider the XRTDC calculated for the luminosity corresponding to the INTEGRAL sensitivity for each object. We considered that IBIS reaches (Paizis et al. 2013) $20 \mathrm{mCrab}(17-60 \mathrm{keV})$ at the $5 \sigma$ level for 1 pointing $(\sim 2 \mathrm{ks})$, and adopted the best Swift broad-band spectra obtained during outburst for each object, to convert from the IBIS band and the $2-10 \mathrm{keV}$ one. These points are also plotted in Fig. 8. The XRT DCs at the INTEGRAL sensitivity are reported in Table 8, Col. 4. They range from $\sim 0.7 \%$ for J17544 to $26 \%$ for J16418, and there is a good match with the corresponding INTEGRAL values for J17544 and J16479. At the INTEGRAL sensitivity J16465 and J17354 have a null DC, so all emission for these sources is below this threshold.

Once the different systematics coming into play in the different definitions of duty cycles are understood, we can once again consider the relationship between the duty cycle and the binary orbital period. We find that the SFXT duty cycles are not clearly correlated with the orbital period. Therefore, wide orbits are not necessarily characterised by low duty cycles, as the clumpy wind models would predict. Instead, an intrinsic mechanism seems to be more likely responsible for the observed variability in SFXTs, i.e., either the wind properties or the compact object properties.
Because it is hard to justify the radically different wind properties in SFXTs from those in normal HMXBs with the same companion spectral type, accretion-inhibition mechanisms seem more plausible, especially in light of the very low DC for J17544 (as well as the other SFXT prototype J1739), for which Bozzo et al. (2008a) interpreted the very broad luminosity ranges observed on timescales as short as hours as transitions across the magnetic and/or centrifugal barriers. This is consistent with the conclusions of Lutovinov et al. (2013) that the flaring behaviour of SFXTs is likely related to the magnetic arrest of their accretion. Alternative mechanisms to partially inhibit accretion in HMXBs have been suggested by Shakura et al. (2012) and applied so far to interpret the low-luminosity regimes of a number of classical supergiant X-ray binaries. The discussion of the applicability of their model to the SFXTs is beyond the scope of the present paper.

\subsection{Differential luminosity distributions}

Other authors (Smith et al. 2012; Paizis \& Sidoli 2014) have used the longer baseline of relatively less sensitive RXTE and INTEGRAL data available-hence geared to best detect the bright flares-to construct cumulative luminosity distributions. In this paper we exploited the higher sensitivity XRT data to construct differential count rate (flux and luminosity) distributions, instead, searching for faint features that originate in different populations of flares in the soft X-ray emission. We have indeed discovered that the SFXT prototypes, J1739 and J17544, as well as 
J16479 and J08408, show two distinct populations of flares. The first one is due to the outburst emission and peaks (or reaches, as in the case of J08408) a few $10^{-9} \mathrm{erg} \mathrm{cm}^{-2} \mathrm{~s}^{-1}$. The second population is due to the out-of-outburst emission, which is characterised by emission spanning up to 4 orders of magnitude in DR (at $100 \mathrm{~s}$ binning). While it is not possible to exclude that particular distributions of the clump and wind parameters may produce a double-peaked differential distribution, this behaviour is more easily explained in terms of different accretion regimes as predicted by the magnetic/centrifugal gating model or the quasi-spherical settling accretion model (Grebenev \& Sunyaev 2007; Bozzo et al. 2008a; Shakura et al. 2012, 2013).

\section{Summary and conclusions}

We have presented the first high-sensitivity (a few $10^{-12}$ erg $\left.\mathrm{cm}^{-2} \mathrm{~s}^{-1}\right)$ soft X-ray $(0.3-10 \mathrm{keV})$ long-term $(\gtrsim 1 \mathrm{yr})$ monitoring with Swift/XRT of three relatively unexplored SFXTs, J08408, J16328, and J16465, which were chosen as those among the SFXT sample, whose hard X-ray duty cycles are the lowest measured. We stress that our monitoring campaigns could only be performed thanks to the extraordinary flexibility in scheduling of Swift, which makes such a monitoring effort cost-effective. Even though the single $1 \mathrm{ks}$ snapshots are shallower than the deep observations of the other pointed observations by XMM-Newton or Suzaku, the advantages are many.

First, thanks to the regular pacing, our data provide a casual sampling of the X-ray light curves at a resolution of $\sim 3-4 \mathrm{~d}$ over $\mathrm{a} \sim 1 \mathrm{yr}$ baseline. They are therefore statistically representative of the long-term properties of these sources that the long looks from other pointed telescopes can only sample, albeit more deeply, only rarely.

Second, these data can be used to measure two defining quantities: i) the dynamical range, fundamental in distinguishing between outbursts of classical supergiant HMXBs $(\lesssim 50)$ and SFXTs ( $\gtrsim 100)$, as described in Negueruela et al. (2006b) and Walter et al. (2006); and ii) the duty cycle as a function of the luminosity across the wide dynamical range spanned by the SFXTs, which is a measure of the activity of each source, and for which different models for emission in SFXTs have contrasting predictions. We can also use the data to perform intensityselected spectroscopy by combining all short exposures, thus reaching the same intrinsic luminosities as those reached by the long looks, and in turn confirming their results.

In this work, we not only created long-term light curves, calculated dynamical ranges and duty cycles, spectroscopically studied the out-of-outburst emission, and created differential luminosity distributions for 3 new sources, but we also compared these properties with those of the remainder of the SFXT sample. Our findings can be summarised as follows:

- All SFXTs share out-of-outburst spectroscopic properties of non-thermal emission plus a soft excess (which becomes increasingly more dominant as the source reaches the lowest emission states) with the general population of supergiant HMXBs. The spectroscopic investigation, therefore, is not an efficient method for distinguishing SFXTs within the HMXB sample.

- The behaviour of J08408 and J16328 resembles that of the SFXT prototypes: the probable X-ray flux is about two orders of magnitude lower than their bright outbursts, accounting for less than $1 \%$ of the total time; the overall dynamical range is DR $\sim 7400$ and $\sim 750$, respectively; the IDC is $\sim 67$ and $61 \%$, respectively, the highest in the SFXTs observed by XRT, consistently with the hard X-ray observations.

- J16465 is to all intents and purposes a persistent source in the XRT, and not an SFXT, with its overall DR $\lesssim 40$ and a duty-cycle of inactivity of $5 \%$.

- By examining the differential luminosity distributions of the SFXT sample, we find that J17544, J1739, J16479, and J08408, show two distinct populations of flares, one due to the outbursts, one due to the out-of-outburst emission, which is characterised by fluxes spanning up to 4 orders of magnitude in DR.

- By exploiting the higher sensitivity afforded by the Swift/XRT observations and by correcting for the sensitivity bias, we find no correlation between the orbital period with any of the duty cycle/activity measurements defined in the soft and hard X-rays. This implies that wide orbits are not characterised by low duty cycles, which answers a longstanding question in SFXT modelling.

- The last two findings can be interpreted in terms of mechanisms that regulate or inhibit accretion, such as a propeller effect, magnetic gating, or hot shells of accreted material above the magnetosphere.

- The definition of duty cycle dramatically depends on the luminosity assumed as lower limit for the calculation.

- Our differential count-rate distributions indicate that, to observe most of the activity of an SFXT, limiting fluxes of at least a decade lower than the sensitivities reached by hard $\mathrm{X}$-ray monitors need to be reached.

Our observations therefore demonstrate that soft X-ray monitoring campaigns on SFXTs, highly variable sources that unpredictably go into outburst, can contribute key ingredients, such as dynamical ranges, duty cycles, and luminosity distributions, towards characterising them among the general HMXB population. In particular, given the $\sim 10^{-12} \mathrm{erg} \mathrm{cm}^{-2} \mathrm{~s}^{-1}$ sensitivity reached in $\sim 1 \mathrm{ks}$ by the XRT, they are uniquely suited to observe most of the activity of an SFXT. They also show that the most effective way to highlight the SFXT nature of a source is combining the soft X-ray inactivity duty cycle and dynamical range.

Finally, we note that, to make significant progress towards understanding SFXTs as a class and within the HMXB context, it is of fundamental importance to continue along this line of investigation, by securing long-term soft X-ray data on more SFXTs. The results of such an investigation will be twofold: on one hand, we will obtain an increased knowledge on a larger number of individual SFXTs, which are on average fainter than the HMXB population and are often located in crowded, heavily absorbed regions of the sky, and therefore have not received adequate attention from lower sensitivity soft X-ray monitors; on the other hand, we can use the combination of soft X-ray inactivity duty cycle and dynamical range to select SFXT candidates among the HMXB population. In this framework, until new insight can be obtained from wide-field, high-sensitivity monitors such as those on board LOFT (Feroci et al. 2012), as recently shown by Bozzo et al. (2013) and Romano et al. (2012a), our monitoring campaigns are the only viable means to reach the low luminosities $\left(L_{2-10 \mathrm{keV}} \sim 10^{33}-10^{34} \mathrm{erg} \mathrm{s}^{-1}\right)$ required to fully characterise the SFXT phenomenology.

Acknowledgements. We wholeheartedly thank the Swift team duty scientists and science planners for their courteous efficiency, and A. Beardmore, M. Capalbi, and H.A. Krimm for helpful discussions. We also thank our referee, Jan-Uwe Ness, for comments that helped improve the paper. P.R. acknowledges contract ASI-INAF I/004/11/0. L.D. thanks Deutsches Zentrum für Luft und Raumfahrt (Grant FKZ 50 OG 1301). 


\section{References}

Barthelmy, S. D., Barbier, L. M., Cummings, J. R., et al. 2005, Space Sci. Rev., 120,143

Bird, A. J., Malizia, A., Bazzano, A., et al. 2007, ApJS, 170, 175

Bozzo, E., Falanga, M., \& Stella, L. 2008a, ApJ, 683, 1031

Bozzo, E., Stella, L., Israel, G., Falanga, M., \& Campana, S. 2008b, MNRAS, 391, L108

Bozzo, E., Stella, L., Ferrigno, C., et al. 2010, A\&A, 519, A6

Bozzo, E., Pavan, L., Ferrigno, C., et al. 2012, A\&A, 544, A118

Bozzo, E., Romano, P., Ferrigno, C., Esposito, P., \& Mangano, V. 2013, Adv. Space Res., 51, 1593

Burrows, D. N., Hill, J. E., Nousek, J. A., et al. 2005, Space Sci. Rev., 120, 165

Clark, D. J., Hill, A. B., Bird, A. J., et al. 2009, MNRAS, 399, L113

Clark, D. J., Sguera, V., Bird, A. J., et al. 2010, MNRAS, 406, L75

Coleiro, A., Chaty, S., Zurita Heras, J. A., Rahoui, F., \& Tomsick, J. A. 2013, A\&A, 560, A108

Corbet, R. H. D., Barthelmy, S. D., Baumgartner, W. H., et al. 2010, ATel, 2588

D’Aì, A., La Parola, V., Cusumano, G., et al. 2011, A\&A, 529, A30

Drave, S. P., Clark, D. J., Bird, A. J., et al. 2010, MNRAS, 409, 1220

Drave, S. P., Bird, A. J., Sidoli, L., et al. 2013, MNRAS, 433, 528

Ducci, L., Sidoli, L., \& Paizis, A. 2010, MNRAS, 408, 1540

Ducci, L., Romano, P., Esposito, P., et al. 2013, A\&A, 556, A72

Elsner, R. F., \& Lamb, F. K. 1977, ApJ, 215, 897

Feroci, M., Stella, L., van der Klis, M., et al. 2012, Exp. Astron., 34, 415

Fiocchi, M., Sguera, V., Bazzano, A., et al. 2010, ApJ, 725, L68

Fiocchi, M., Bazzano, A., Bird, A. J., et al. 2013, ApJ, 752, 19

Gehrels, N., Chincarini, G., Giommi, P., et al. 2004, ApJ, 611, 1005

Götz, D., Schanne, S., Rodriguez, J., et al. 2006, ATel, 813, 1

Grebenev, S. A., \& Sunyaev, R. A. 2007, Astron. Lett., 33, 149

Grupe, D., Kennea, J., Evans, P., et al. 2009, ATel, 2075, 1

Hickox, R. C., Narayan, R., \& Kallman, T. R. 2004, ApJ, 614, 881

Hill, J. E., Burrows, D. N., Nousek, J. A., et al. 2004, in X-Ray and Gamma-

Ray Instrumentation for Astronomy XIII, eds. K. A. Flanagan, \& O. H. W.

Siegmund, Proc. SPIE, 5165, 217

in't Zand, J. J. M. 2005, A\&A, 441, L1

Kalberla, P. M. W., Burton, W. B., Hartmann, D., et al. 2005, A\&A, 440, 775

Kennea, J. A., \& Campana, S. 2006, ATel, 818, 1

Kreykenbohm, I., Wilms, J., Kretschmar, P., et al. 2008, A\&A, 492, 511

Krimm, H. A., Holland, S. T., Corbet, R. H. D., et al. 2013, ApJS, 209, 14

Krivonos, R., Tsygankov, S., Lutovinov, A., et al. 2012, A\&A, 545, A27

La Parola, V., Cusumano, G., Romano, P., et al. 2010, MNRAS, 405, L66

Levine, A. M., \& Corbet, R. 2006, ATel, 940, 1

Leyder, J.-C., Walter, R., Lazos, M., Masetti, N., \& Produit, N. 2007, A\&A, 465, L35

Liu, Q. Z., van Paradijs, J., \& van den Heuvel, E. P. J. 2005, A\&A, 442, 1135

Liu, Q. Z., van Paradijs, J., \& van den Heuvel, E. P. J. 2006, A\&A, 455, 1165

Lutovinov, A., Revnivtsev, M., Gilfanov, M., et al. 2005, A\&A, 444, 821

Lutovinov, A. A., Revnivtsev, M. G., Tsygankov, S. S., \& Krivonos, R. A. 2013, MNRAS, 431, 327
Masetti, N., Bassani, L., Bazzano, A., et al. 2006, ATel, 815, 1

Mereghetti, S., Sidoli, L., Paizis, A., \& Gotz, D. 2006, ATel, 814, 1

Morris, D. C., Smith, R. K., Markwardt, C. B., et al. 2009, ApJ, 699, 892

Negueruela, I., Smith, D. M., Harrison, T. E., \& Torrejón, J. M. 2006a, ApJ, 638, 982

Negueruela, I., Smith, D. M., Reig, P., Chaty, S., \& Torrejón, J. M. 2006b, 604, 165

Negueruela, I., Smith, D. M., Torrejón, J. M., \& Reig, P. 2007, ESA SP, 622, 255

Negueruela, I., Torrejón, J. M., Reig, P., Ribó, M., \& Smith, D. M. 2008, AIP Conf. Proc. (Elsevier), 1010, 252

Nespoli, E., Fabregat, J., \& Mennickent, R. E. 2008, A\&A, 486, 911

Paizis, A., \& Sidoli, L. 2014, MNRAS

Paizis, A., Mereghetti, S., Götz, D., et al. 2013, Astronomy and Computing, 1, 33

Rahoui, F., Chaty, S., Lagage, P.-O., \& Pantin, E. 2008, A\&A, 484, 801

Rampy, R. A., Smith, D. M., \& Negueruela, I. 2009, ApJ, 707, 243

Romano, P., Campana, S., Chincarini, G., et al. 2006, A\&A, 456, 917

Romano, P., Sidoli, L., Mangano, V., Mereghetti, S., \& Cusumano, G. 2007, A\&A, 469, L5

Romano, P., Sidoli, L., Cusumano, G., et al. 2009, MNRAS, 399, 2021

Romano, P., Sidoli, L., Ducci, L., et al. 2010, MNRAS, 401, 1564

Romano, P., La Parola, V., Vercellone, S., et al. 2011, MNRAS, 410, 1825

Romano, P., Bozzo, E., Esposito, P., Ferrigno, C., \& Mangano, V. 2012a, in AIP Conf. Proc. 1505, eds. F. A. Aharonian, W. Hofmann, \& F. M. Rieger, 813

Romano, P., Mangano, V., Ducci, L., et al. 2012b, MNRAS, 419, 2695

Romano, P., Lien, A. Y., Evans, P. A., et al. 2013a, ATel, 5190, 1

Romano, P., Mangano, V., Ducci, L., et al. 2013b, Adv. Space Res., 52, 1593

Romano, P., Mangano, V., Ducci, L., et al. 2013c, Mem. Soc. Astron. It., 84, 602

Romano, P., Krimm, H. A., Palmer, D. M., et al. 2014, A\&A, 562, A2

Sguera, V., Barlow, E. J., Bird, A. J., et al. 2005, A\&A, 444, 221

Shakura, N., Postnov, K., Kochetkova, A., \& Hjalmarsdotter, L. 2012, MNRAS, 420, 216

Shakura, N., Postnov, K., \& Hjalmarsdotter, L. 2013, MNRAS, 428, 670

Sidoli, L., Romano, P., Mereghetti, S., et al. 2007, A\&A, 476, 1307

Sidoli, L., Romano, P., Mangano, V., et al. 2008, ApJ, 687, 1230

Smith, D. M., Markwardt, C. B., Swank, J. H., \& Negueruela, I. 2012, MNRAS, 422, 2661

Tomsick, J. A., Chaty, S., Rodriguez, J., Walter, R., \& Kaaret, P. 2009, ApJ, 701, 811

Torrejón, J. M., Negueruela, I., Smith, D. M., \& Harrison, T. E. 2010, A\&A, 510, A61

Ubertini, P., Lebrun, F., Di Cocco, G., et al. 2003, A\&A, 411, L131

van der Meer, A., Kaper, L., di Salvo, T., et al. 2005, A\&A, 432, 999

Vaughan, S., Goad, M. R., Beardmore, A. P., et al. 2006, ApJ, 638, 920

Walter, R., \& Zurita Heras, J. 2007, A\&A, 476, 335

Walter, R., Zurita Heras, J., Bassani, L., et al. 2006, A\&A, 453, 133

Zurita Heras, J. A., \& Walter, R. 2004, ATel, 336, 1 